\title{
Donor-Acceptor-Based Poly(toluidine-co-chloroaniline)s: Synthesis and Characterization
}

\author{
P. SAVITHA, D. N. SATHYANARAYANA \\ Department of Inorganic and Physical Chemistry, Indian Institute of Science, Bangalore 560012, India
}

\begin{abstract}
A series of poly(o- $/ m$-toluidine-co-o- $/ m$-chloroaniline $)$ copolymers of different compositions were synthesized by an emulsion method with ammonium persulfate as the oxidant. The conductivity of the copolymers was two to five orders of magnitude higher than that of the homopolymers poly(o-toluidine) and poly( $m$-chloroaniline). Among the copolymers, the copolymer of $o$-toluidine and $m$-chloroaniline exhibited a maximum conductivity of $0.14 \mathrm{~S} \mathrm{~cm}^{-1}$. The conductivity of these copolymers was also higher than that of poly(aniline-co-chloroaniline). The properties of the copolymers were greatly influenced by the positions of the substituents and the concentrations of the individual monomers in the feed. All the copolymers were completely soluble in polar solvents such as dimethyl sulfoxide and showed higher heat stability as the chloroaniline concentration increased. These effects could be interpreted in terms of extensive hydrogen bonding and interchain linking and, therefore, higher electron delocalization in these copolymers due to the presence of electron-rich toluidine rings adjacent to electron-deficient chloroaniline.
\end{abstract}

Keywords: conducting polymers; copolymerization; emulsion polymerization; poly(o-/m-chloroaniline); poly $(o-/ m$-toluidine $)$

\section{INTRODUCTION}

Polyaniline (PANI) has attracted attention because of its interesting chemical, electrochemical, and optical properties, which can be controlled by doping with protonic acids or by treatments with oxidants such as iodine. ${ }^{1}$ Applications of PANI, such as electrodes in secondary batteries, active materials in electrochromic display devices, and corrosion inhibitors, have been reported. ${ }^{2}$ However, these applications have been limited because of the poor solubility of PANI in common organic solvents. Efforts have been made to im-

Correspondence to: D. N. Sathyanarayana (E-mail: dns@ ipc.iisc.ernet.in) prove the solubility and electronic properties of PANI with substituted PANI with substituents such as alkyl, ${ }^{3}$ alkoxy ${ }^{4}$ alkoxy sulfonyl,${ }^{5}$ and carboxylic acid groups ${ }^{6}$ to the monomer. In this context, note that the homopolymerization of $o$-chloroaniline and $m$-chloroaniline is rather difficult. This is ascribed to the electron-withdrawing effect of the chlorine substituent. The homopolymerization of chloroanilines has been accomplished chemically with stronger oxidizing agents such as chromic acid and sodium dichromate. Polychloroanilines exhibit very low conductivity of approximately $10^{-6}-10^{-8} \mathrm{~S} / \mathrm{cm}$, and this has been ascribed to the more electronegative group on the phenyl ring. ${ }^{7}$

Copolymerization offers a way of improving the processability of PANI. The copolymerization of aniline with derivatives with different types of 
substituents, such as alkyl, ${ }^{8,9}$ alkoxy, ${ }^{10}$ carboxylic acid, ${ }^{11}$ and sulfonic acid groups, ${ }^{12,13}$ has been carried out to improve the processibility of PANI.

There are a few reports supporting the use of alternating donor-acceptor moieties in the polymer chain to increase the intrinsic conductivity of the polymers by virtue of decreased band gaps. ${ }^{14,15}$ Such systems are predicted to have broader valence and conduction bands leading to smaller band gaps. Polymers with both the donor and acceptor moieties on the same molecule and on different molecules have also been investigated. ${ }^{16,17}$

We report in this work the chemical synthesis of $\operatorname{poly}(o-/ m$-toluidine-co-o- $/ m$-chloroaniline $)$ s with various molar ratios of the comonomers in the feed with the aim of understanding the effect of the presence of both the highly electron-withdrawing halogen atom and the electron-donating methyl group substituent in the copolymer. Because the size of the $-\mathrm{CH}_{3}$ group is nearly the same as that of the $-\mathrm{Cl}$ group, the variation in the properties of the copolymers from that of the homopolymer poly(o-toluidine) (POT) or $\operatorname{poly}(m$ toluidine) (PMT) may result chiefly from the electronic effects of substitution. Emulsion polymerization has been used for the synthesis of copolymers. This method helps to increase the interactions between the oxidant, dopant, and monomers because the reaction occurs in a large number of loci dispersed in a continuous phase providing a larger surface area for the polymerization to take place. ${ }^{18}$ The structure and properties induced by copolymerization are discussed with various spectroscopic, thermal, morphological, and electrical measurements. The interesting features of these new copolymers prepared with various molar compositions of the comonomers with electron-donating and electron-withdrawing substituents are their high solubility in polar solvents and good conductivity. Their compositions have been determined with Fourier transform infrared (FTIR) spectroscopy.

\section{EXPERIMENTAL}

\section{Materials and Methods}

$m$-Chloroaniline and $o$ - $/ m$-toluidines (Merck) were distilled under reduced pressure before use. $o$-Chloroaniline (Aldrich) and all other chemicals were used as received.
The ultraviolet-visible (UV-vis) absorption spectra of the copolymer solutions in dimethyl sulfoxide (DMSO) were recorded on a Hitachi U-3000 spectrophotometer. The IR spectra of the solid samples were recorded on a Bruker IFS 55 FTIR spectrometer as KBr pellets. Fourier transform Raman (FT-Raman) spectra were recorded on a Bruker RFS-100/S spectrometer for the powder samples with an $\mathrm{Nd}^{3+}$ :YAG laser with a laser power of $22 \mathrm{~mW}$ at the samples with $1064 \mathrm{~nm}$ for excitation. ${ }^{1} \mathrm{H}$ NMR spectra were obtained on a Bruker AMX 400-MHz spectrometer with tetramethylsilane as an internal reference and DMSO- $d_{6}$ as a solvent. The conductivity of the copolymers was measured by the four-probe method (pressure contact) on pressed pellets obtained by subjecting the powder to a pressure of $50 \mathrm{kN}$. The error in the resistance measurements under galvanostatic conditions with a Keithley 220 programmable current source and a Keithley 195A digital voltammeter was less than $2 \%$. The thermogravimetric analysis (TGA) measurements were performed with a Mettler-Toledo Star system at a heating rate of $10{ }^{\circ} \mathrm{C} / \mathrm{min}$ in static air. The scanning electron microscopy (SEM) measurements were made with a JEOL JSM $5600 \mathrm{~L}$ scanning electron microscope. The $\mathrm{X}$-ray diffraction (XRD) patterns were recorded for the powdered materials with a Siemens D5005 $\mathrm{X}$-ray diffractometer.

\section{Chemical Copolymerization}

A typical procedure for the synthesis of a copolymer with a 1:1 molar composition of the monomers was as follows. Chloroform $(100 \mathrm{~mL})$ was taken, and an aqueous solution of $0.1 \mathrm{M}$ sodium lauryl sulfate, the emulsifier $(2.25 \mathrm{~g}$ in $50 \mathrm{~mL}$ of water), was added to it slowly with constant stirring; a milky white emulsion was obtained. Toluidine $(1.1 \mathrm{~mL}, 0.05 \mathrm{M})$ and chloroaniline $(1.1 \mathrm{~mL}$, $0.05 \mathrm{M}$ ) were added to it followed by the dropwise addition of $50 \mathrm{~mL}$ of the dopant $\mathrm{HCl}(1 \mathrm{M})$ and 50 $\mathrm{mL}$ of an aqueous solution of the oxidant (ammonium persulfate; $5.7 \mathrm{~g}, 0.1 \mathrm{M}$ ). The polymerization was allowed to continue with stirring for $24 \mathrm{~h}$ at the ambient temperature. The organic phase was separated and washed repeatedly with water. It was then added to $600 \mathrm{~mL}$ of acetone (nonsolvent) to precipitate the copolymer. After $10 \mathrm{~h}$, the greenish precipitate was filtered under suction and washed with an excess of acetone to remove the oligomers and the oxidant. The copolymer salt was dried in vacuo for $72 \mathrm{~h}$. 


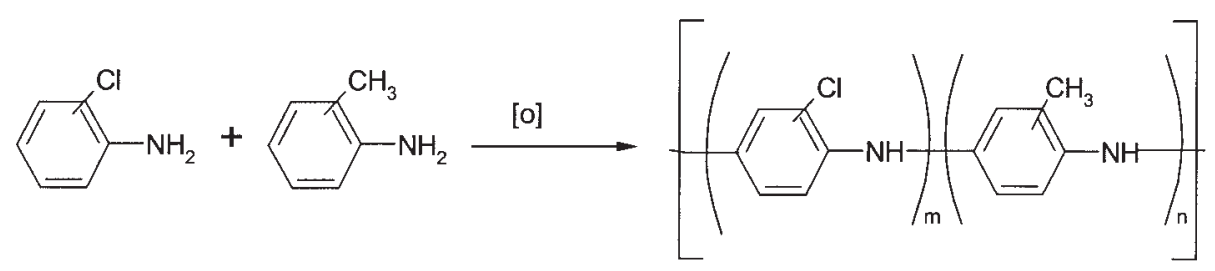

Scheme 1 Oxidative copolymerization of toluidine with chloroaniline.

\section{RESULTS AND DISCUSSION}

The copolymers poly(o-toluidine-co-o-chloroaniline) and poly( $m$-toluidine-co- $m$-chloroaniline) were synthesized by chemical copolymerization from the respective chloroaniline and toluidine comonomers in four different molar feed compositions (3:1, 2:1, 1:1, and 1:2). However, poly(o-toluidine-co- $m$-chloroaniline) and $\operatorname{poly}(m$-toluidine-co-o-chloroaniline) copolymers were synthesized with only the 1:1 comonomer feed ratio. The polymerization reaction is shown in Scheme 1.

The nomenclature for the copolymers follows the monomers and their comonomer feed ratios used for the synthesis. For example, OTOC31 refers to a copolymer of $o$-toluidine (OT) and $o$ chloroaniline (OC) for which the comonomer feed ratio of $o$-toluidine to $o$-chloroaniline was $3: 1$. Similarly, for the synthesis of copolymer MTMC12, a comonomer molar feed ratio of 1:2 of $m$-toluidine to $m$-chloroaniline was used. POT and PMT are the homopolymers of $o$-toluidine and $m$-toluidine, respectively.

\section{Yield and Conductivity}

The yield of the copolymers (Table 1) decreased with increasing chloroaniline content in the comonomer feed. This could be traced to the electron-withdrawing effect of the substituent $-\mathrm{Cl}$ group, which lowered the amine basicity and led to a lower degree of polymerization. The $m$-toluidine/chloroaniline copolymers were obtained in lower yields in comparison with those of $o$-toluidine with chloroaniline. The $-\mathrm{CH}_{3}$ group in the meta position might have been less effective in increasing the electron density along the chain than the ortho substituent.

The electrical conductivity of the copolymers (Table 1) was higher than that of the homopolymers polytoluidine and polychloroaniline. The conductivity of the copolymers followed the trend of an initial small increase and then a decrease with an increase in the amount of chloroaniline in the feed. The donor-acceptor interactions being dependent on the copolymer compositions, a higher $o$-chloroaniline or $o$-toluidine content in the feed could lead to comparatively lower electron delocalization. Also, a high chloroaniline content in the comonomer feed could reduce the donor-acceptor interaction and result in reduced conductivity. Copolymer OTMC11 had the highest conductivity $\left(0.14 \mathrm{~S} \mathrm{~cm}^{-1}\right)$ of all the copolymers when the substituent groups $-\mathrm{CH}_{3}$ and $-\mathrm{Cl}$ were present in the ortho and meta posi-

Table 1. Yields, Compositions, Conductivity, and Absorption Bands for Toluidine-Chloroaniline Copolymers

\begin{tabular}{lccccccc}
\hline Copolymer & $\begin{array}{c}\text { Composition } \\
\left(\mathrm{M}_{1}: \mathrm{M}_{2}\right)^{\mathrm{a}}\end{array}$ & $\begin{array}{c}\text { Yield } \\
(\%)\end{array}$ & $\begin{array}{c}\text { Conductivity } \\
\left(10^{-3} \mathrm{~S} / \mathrm{cm}\right)\end{array}$ & \multicolumn{4}{c}{$\begin{array}{c}\text { Absorption Bands } \\
(\text { in DMSO; } \lambda \mathrm{nm})\end{array}$} \\
\hline OTOC31 & $1.7: 1$ & 95 & 4.5 & 322 & 448 & 621 & 900 \\
OTOC21 & $0.7: 1$ & 44 & 6.9 & 311 & 448 & 615 & 777 \\
OTOC11 & $0.66: 1$ & 37 & 5.1 & 311.5 & 446 & 609 & 787 \\
OTOC12 & $0.82: 1$ & 12 & 2.3 & 312 & 424 & 613 & 805 \\
OTMC11 & $1.06: 1$ & 34 & 141 & 297 & 415 & 588 & 807 \\
MTMC31 & - & 33 & 0.4 & 298 & 414 & 586 & - \\
MTMC21 & $1.5: 1$ & 27 & 7.2 & 299 & 413 & 589 & - \\
MTMC11 & $1.32: 1$ & 27 & 2.6 & 298 & 415 & 594 & - \\
MTMC12 & $2.17: 1$ & 3 & - & 299 & 400 & 588 & - \\
MTOC11 & $1.6: 1$ & 24 & 0.6 & 312 & 423 & 596 & 818 \\
\hline
\end{tabular}

${ }^{\mathrm{a}} \mathrm{M}_{1}=\mathrm{OT} / \mathrm{MT} ; \mathrm{M}_{2}=\mathrm{OC} / \mathrm{MC}$. 
tions, respectively. Interestingly, the composition determined for this copolymer was 1:1 of o-toluidine: $m$-chloroaniline (Table 1 ).

\section{Absorption Spectra}

Figure 1 shows the UV-vis spectra of copolymers OTOC21 and MTOC21 in their doped form. The absorption bands of the copolymers are listed in Table 1 . The spectrum of $o$-chloroaniline in DMSO exhibits a broad band around $301 \mathrm{~nm}$, whereas that of $m$-chloroaniline has a band at $299 \mathrm{~nm}$. The spectrum of homopolymer POT in the emeraldine salt form consists of four bands at $311,443,608$, and $790 \mathrm{~nm} .{ }^{19}$ The absorption spectrum of the PMT-HCl salt is similar, with the bands located at $303,413,587$, and $859 \mathrm{~nm} .{ }^{19}$

The spectra of all the copolymers also generally display four absorption bands. The first one at $298-322 \mathrm{~nm}$ is assigned to the $\pi-\pi^{*}$ transition of the benzenoid rings on the basis of earlier studies. ${ }^{20}$ It is related to the extent of conjugation between the phenyl rings along the polymer chain. A bathochromic shift of the 311-nm band of POT for copolymer OTOC31 implies an increase in the extent of conjugation. This bathochromic shift decreases as the $o$-chloroaniline content in the copolymer increases from 37 to $59 \%$ (OTOC31 and OTOC21, respectively) and remains unchanged with a further increase in the o-chloroaniline content. Copolymer OTMC11, however, shows a hypsochromic shift for this band in comparison with OTOC11. This may be expected because the substituent group - $\mathrm{Cl}$ in the meta position to the amino group reduces the electron density to a greater extent than the $-\mathrm{Cl}$ substitu-

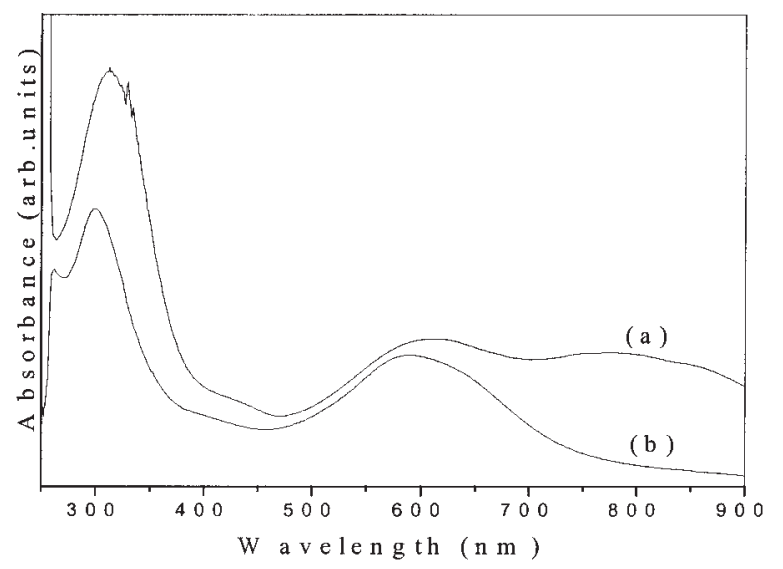

Figure 1. UV-vis spectra of (a) OTOC21 and (b) MTOC21. ent in the ortho position. The copolymers of $m$ toluidine with $m$-chloroaniline show a small hypsochromic shift for this band in comparison with the copolymers of $o$-toluidine with $o$-chloroaniline and homopolymer PMT. This again may be due to the highly electron-withdrawing effect of the $-\mathrm{Cl}$ group in the meta position and the lower + I effect of the methyl group in the meta position for these copolymers.

The third absorption band at 588-620 $\mathrm{nm}$ is assigned to the quinoid ring transition (charge transfer from the highest occupied molecular orbital of the benzenoid ring to the lowest unoccupied molecular orbital of the quinoid ring). ${ }^{21-23} \mathrm{It}$ is sensitive to the overall oxidation state of the polymer. This band also shows bathochromic shifts for all the $o$-toluidine copolymers except OTMC11. This peak shows very little shifting for $m$-toluidine copolymers with $m$-chloroaniline in comparison with homopolymer PMT, except for MTOC11; this reveals the effect of the position and the concentration of toluidines with the donor $-\mathrm{CH}_{3}$ group and chloroaniline with the acceptor - Cl group on the degree of oxidation. The remaining two bands at $410-450$ and $790-880 \mathrm{~nm}$ are due to the polaronic transitions. ${ }^{24}$ The polaronic bands are found only in the spectrum of the salt, and their presence indicates the greater solubility of the copolymer salt in the solvent used to measure the spectra because the intensity of these absorption bands is very low. The $m$-toluidine copolymers with $m$-chloroaniline do not show the $800-\mathrm{nm}$ peak.

\section{FTIR Spectra}

The FTIR spectra of OTOC31 and OTOC11 are shown in Figure 2 and are typical of the copolymers. A small, weak band at approximately $3200 \mathrm{~cm}^{-1}$ in the spectrum of POT, corresponding to the hydrogen-bonded $\mathrm{N}-\mathrm{H}$ vibrations, is shifted to $3215 \mathrm{~cm}^{-1}$ in the copolymers. Twin bands centered around 2920 and $2850 \mathrm{~cm}^{-1}$ can be assigned to the aliphatic $\mathrm{C}-\mathrm{H}$ stretching of the toluidine segment. The characteristic bands of the emeraldine form of POT occur at 1587, 1491, $1381,1315,1213,1153,1109$, and $808 \mathrm{~cm}^{-1}$, whereas those of PMT occur at 1585, 1489, 1369, 1322, 1211, 1151, 1107, and $806 \mathrm{~cm}^{-1}$. These peaks are shifted in the spectra of the copolymers and confirm the presence of $o-/ m$-toluidine units in the copolymers. For example, the spectrum of OTOC11 exhibits two main bands at 1589 and $1483 \mathrm{~cm}^{-1}$ assigned to the ring stretching vibra- 


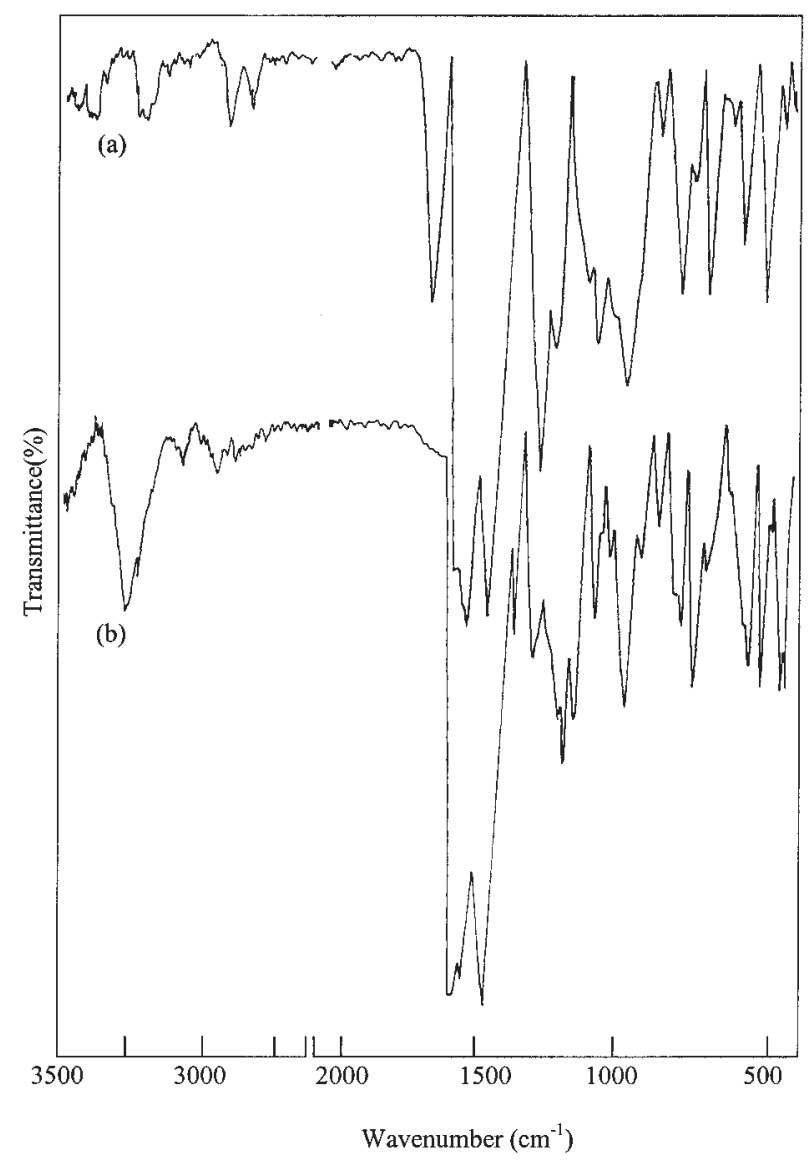

Figure 2. FTIR spectra of (a) OTOC31 and (b) OTOC11.

tions of the quinoid and benzenoid rings, respectively. The former band is unaffected whereas the latter band is found at $1473 \mathrm{~cm}^{-1}$ in the spectrum of MTMC11. A medium-intensity peak at 1300$1325 \mathrm{~cm}^{-1}$ is due to the $\mathrm{C}-\mathrm{N}$ stretching vibration in alternate units of quinoid-benzenoid-quinoid rings, whereas the band at $1375-1381 \mathrm{~cm}^{-1}$ corresponds to the $\mathrm{C}=\mathrm{N}^{+}$stretching vibration. The $1170-1190-\mathrm{cm}^{-1}$ absorption in the spectra of the copolymers can be assigned to a vibrational mode of a $\mathrm{B}-\mathrm{NH}^{+}=\mathrm{Q}$ structure, which is formed upon protonation. It indicates the existence of positive charges in the chain and the distribution of the dihedral angle between the quinoid and benzenoid rings. It is known to increase in intensity with the degree of doping in the polymer backbone. ${ }^{25}$ The bands become more intense because of the enhancement of the oscillator strengths of the backbone-related vibrations by coupling with the proton-induced charge. A band near $1107 \mathrm{~cm}^{-1}$ is assigned to $\mathrm{C}-\mathrm{H}$ in-plane bending. This normally IR-inactive mode becomes active when the protonation process induces conformational changes in the polymer chain through the formation of polarons or bipolarons. The spectra of the copolymers exhibit a fairly intense absorption at $758-764 \mathrm{~cm}^{-1}$ due to the characteristic C- - l stretching; it occurs as a strong absorption at $746 \mathrm{~cm}^{-1}$ in the monomer and indicates the presence of chloroaniline units in the copolymers.

The intense $\mathrm{C}-\mathrm{H}$ out-of-plane bending vibrations of the 1,2,4-trisubstituted benzene rings appear around 880 and $810 \mathrm{~cm}^{-1}$ and indicate that the monomers in the copolymers are bonded head to tail in agreement with the expected structures. The IR spectra of the $o$-toluidine and $o$-chloroaniline copolymers show the quinoid and benzenoid absorptions at about 1580 and $1495 \mathrm{~cm}^{-1}$ for the doped structure. These bands occur at 1590 and $1480 \mathrm{~cm}^{-1}$ for $m$-toluidine $/ m$-chloroaniline copolymers. The IR spectra of the copolymers show the characteristic band of the functional group ${ }^{26}$ $-\mathrm{CH}_{3}$ at about $1005 \mathrm{~cm}^{-1}$.

The compositions of the copolymers were determined by a comparison of the characteristic bands of the functional groups of toluidine and chloroaniline found at 1005 and $758 \mathrm{~cm}^{-1}$, respectively, in the copolymers (Table 1 ). The intensity of the $758-\mathrm{cm}^{-1}$ peak increases with increasing chloroaniline content in the comonomer feed. o-Chloroaniline copolymerized to a greater degree than $m$ chloroaniline (Table 1). This could be due to the greater $-\mathrm{I}$ effect of the $-\mathrm{Cl}$ substituent in the meta position.

\section{FT-Raman Spectra}

The Raman spectra support the formation of copolymers as deduced from the IR spectra. The Raman band at $1592 \mathrm{~cm}^{-1}$ is attributed to the $\mathrm{C}=\mathrm{C}$ ring stretching vibration of the quinoid ring, and that at $1513 \mathrm{~cm}^{-1}$ is attributed to the $\mathrm{C}=\mathrm{C}$ ring stretching vibration of the benzenoid ring. The $1346-$ and $1263-\mathrm{cm}^{-1}$ bands are assigned to the $\mathrm{N}-\mathrm{H}$ and $\mathrm{C}-\mathrm{H}$ in-plane bending modes, respectively. A band at $1326 \mathrm{~cm}^{-1}$ is assigned to the $\mathrm{C}$ (ring) $-\mathrm{N}$ stretching mode. These bands, which are also found in the FT-Raman spectra of homopolymers PMT and POT, confirm the presence of POT and PMT segments in the copolymer. $o$-Chloroaniline shows intense bands at $1025,838,680$, and $563 \mathrm{~cm}^{-1}$ and weak bands at 1616,1309 , and $474 \mathrm{~cm}^{-1}$. In the copolymers, these bands occur around 1618, 1309, 1031, and $570 \mathrm{~cm}^{-1}$. The corresponding $m$-chloroaniline bands occur at 1614, 1311, 1045, and $576 \mathrm{~cm}^{-1}$. 
Some of the chief bands of POT and PMT appear to be split in the Raman spectra because the spectra of chloroanilines have bands in almost the same region as that of POT or PMT. As noted earlier from the IR spectra, the intensity of the peaks of chloroaniline increases with increasing chloroaniline content in the comonomer feed, qualitatively revealing the composition of the copolymer. The bands arising from the toluidine units in the copolymers undergo a shift to lower frequencies in comparison with those of homopolymer POT or PMT, and they show an increase in the conjugation, as deduced from the bathochromic shift of the peaks in the UV-vis and IR spectra.

\section{${ }^{1} \mathrm{H}$ NMR Spectra}

Figure 3 shows the ${ }^{1} \mathrm{H}$ NMR spectra of copolymers OTOC11 and OTMC11. The ${ }^{1} \mathrm{H}$ NMR spectra of the copolymers in DMSO- $d_{6}$ solutions show the peaks of the protons of the methyl group on the benzenoid ring arising from the toluidine seg- ments in the region of $\delta 1.8-2.1 \mathrm{ppm}$, whereas the protons of the methyl group on quinoid rings (expected at $\delta 1.1-1.3 \mathrm{ppm}$ ) are masked by the surfactant impurities. The $-\mathrm{NH}$ proton resonances are found at $3.6 \mathrm{ppm}$. The resonance signals from 6.3 to $7.5 \mathrm{ppm}$ correspond to aromatic protons (which appear at 6.3-6.8 ppm for o-chloroaniline and at 6.9-7.4 ppm for homopolymer POT). The peaks due to aromatic protons of $o$-chloroaniline, which occur at $\delta 6.4-7.1 \mathrm{ppm}$ in the monomer, are shifted upfield by $0.1-0.3 \mathrm{ppm}$, whereas those of toluidine, which occur around 6.9-7.4 ppm in the homopolymers, undergo a slight downfield shift of 0.1-0.5 ppm. This reveals that the electron density of the chloroaniline segment of the copolymer is increased in comparison with that of the monomer, whereas the electron density of the toluidine units is reduced because of the proximity of the rings to the electron-withdrawing chlorine.

The ${ }^{1} \mathrm{H}$ NMR spectra are informative for obtaining the compositions of the copolymers. The integrated intensities of the peaks due to the aromatic protons of toluidines and chloroanilines
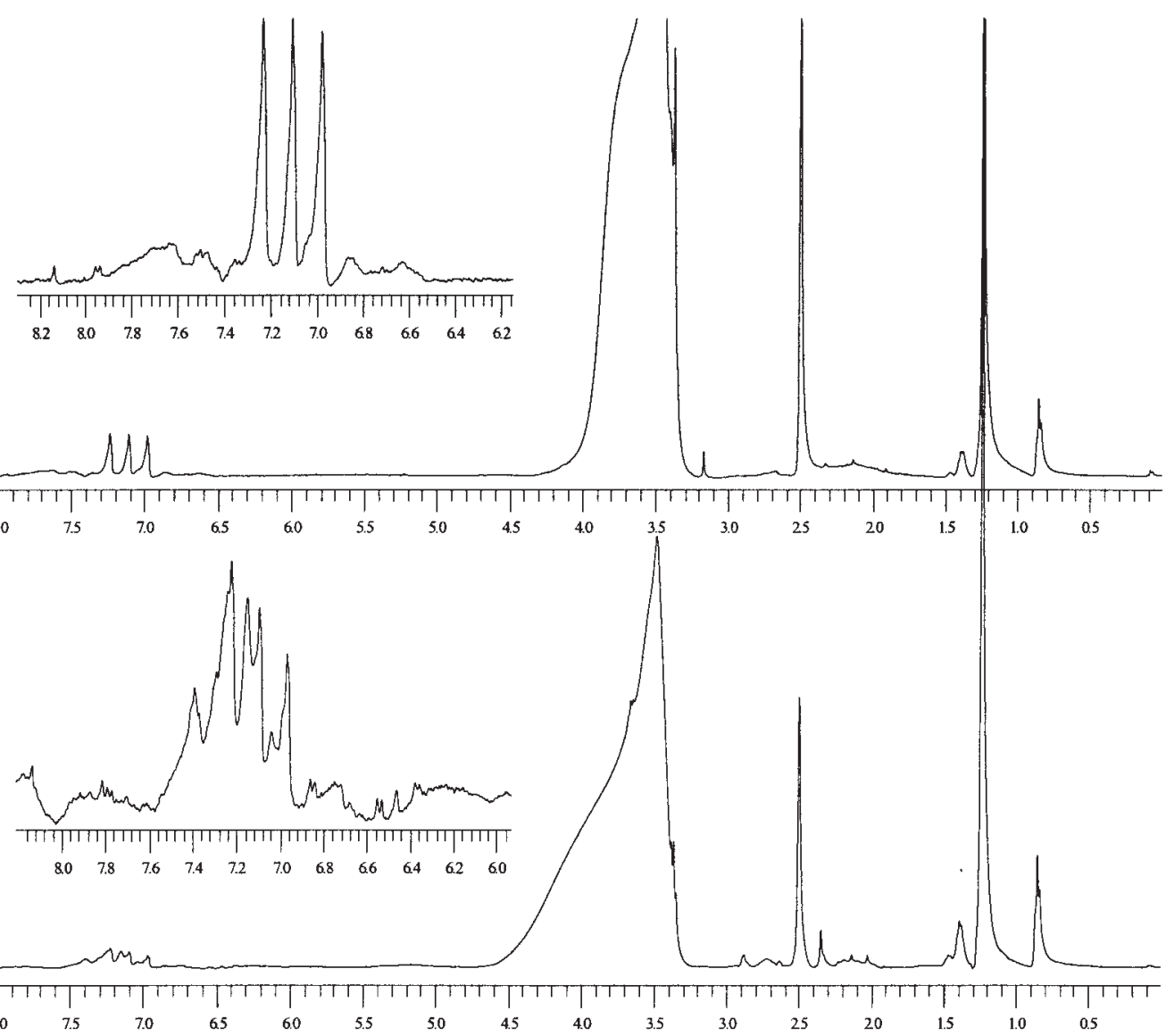

(b)

(a)

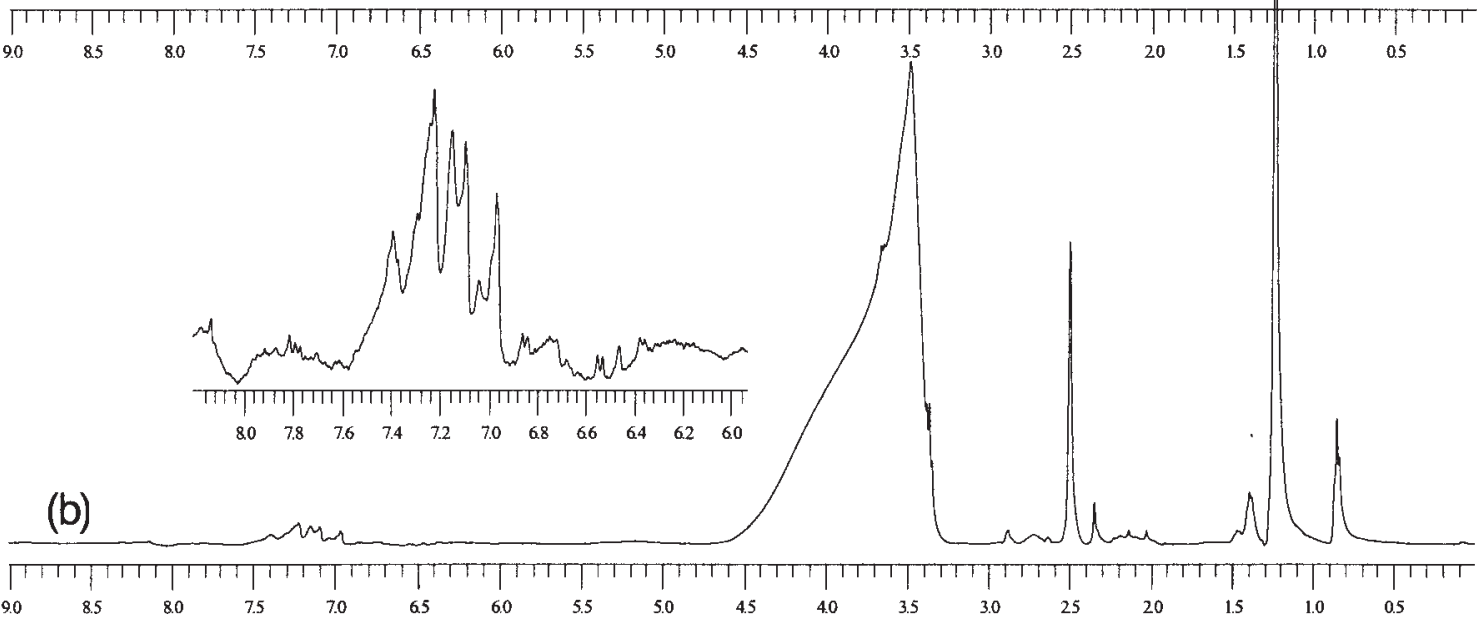

Figure 3. ${ }^{1} \mathrm{H}$ NMR spectra of (a) OTOC11 and (b) OTMC11. 
qualitatively provide the proportions of the toluidine and chloroaniline units in the copolymer. Almost all the copolymers have high concentrations of $m$-toluidine according to the spectral intensities, regardless of their comonomer feed compositions. However, the spectrum of copolymer OTMC11 shows almost equal intensities of the resonance peaks for the aromatic protons of $o^{-}$ toluidine and $m$-chloroaniline.

The ${ }^{1} \mathrm{H}$ NMR spectra also provide information about the relative reactivities of the two monomers. The signals of the $o$-chloroaniline segments have a lower intensity than the $m$-chloroaniline segments. The $m$-toluidine content in a given copolymer is much higher than the $o$-toluidine content in the corresponding copolymer. For example, OTOC11 had a much lower concentration of $o$-toluidine than the concentration of $m$-toluidine in MTOC11. The same observation was made for copolymers OTMC11 and MTMC11. The decreasing yield with a higher reactivity of $m$-toluidine, in comparison with that of o-toluidine, is also supported by the yield of the corresponding homopolymers ${ }^{26}$ and the copolymers.

\section{TGA}

The weight-loss patterns in the thermogravimetric curves of the copolymers follow earlier reports on homopolymers POT and PMT. ${ }^{27,28}$ The first weight-loss step, from 50 to $140{ }^{\circ} \mathrm{C}$, corresponds to a loss of moisture, volatilization of the solvent, and adsorbed $\mathrm{HCl}$. The next step, from 150 to $300{ }^{\circ} \mathrm{C}$, is due to the loss of dopant. The final weight-loss step, occurring between 400 and $600{ }^{\circ} \mathrm{C}$, corresponds to the degradation of the copolymers.

The thermal stability was higher for the copolymer obtained with $o$-chloroaniline in the comonomer feed (Table 2). For example, when the $o$-chloroaniline content in the feed was varied from 0.033 to $0.05 \mathrm{M}$ (yielding copolymers OTOC21 and OTOC11, respectively) the degradation temperature increased from 373 to $413{ }^{\circ} \mathrm{C}$. This suggests interchain linking and hydrogen bonding in these copolymers due to the presence of the substituent - $\mathrm{Cl}$ group of o-chloroaniline, and more energy was required to disrupt these bonds. The copolymers of $o$-toluidine with $o$-chloroaniline apparently possessed greater thermostability than the copolymers of $o$-toluidine with $m$-chloroaniline. For example, the degradation temperatures of OTOC11 and OTMC11 were 413 and $398{ }^{\circ} \mathrm{C}$, respectively. Similarly, the copolymer
Table 2. Thermal Analysis Data for Selected Toluidine-Chloroaniline Copolymers

\begin{tabular}{|c|c|c|c|}
\hline \multirow[b]{2}{*}{ Sample } & \multicolumn{3}{|c|}{$\begin{array}{l}\text { Temperature Range }\left({ }^{\circ} \mathrm{C}\right) \\
\text { [Weight Loss }(\%)]\end{array}$} \\
\hline & $\begin{array}{l}\text { First } \\
\text { Step }\end{array}$ & $\begin{array}{l}\text { Second } \\
\text { Step }\end{array}$ & $\begin{array}{l}\text { Third } \\
\text { Step }\end{array}$ \\
\hline OTOC21 & 50-119 (8) & $150-287(21)$ & 373 \\
\hline отоC11 & $50-138(8)$ & $187-300$ & 413 \\
\hline OTMC11 & $50-138(6)$ & $177-313(21)$ & 398 \\
\hline MTMC11 & $50-119$ (4) & $173-315$ & 387 \\
\hline
\end{tabular}

of $m$-toluidine and $m$-chloroaniline, MTMC11, had lower thermal stability than both OTOC11 and OTMC11. This may have been due to the lower electron-donating ability of the $-\mathrm{CH}_{3}$ group in the $m$-position ( $m$-toluidine) and the higher electron-withdrawing effect of $-\mathrm{Cl}$ in the $m$-position ( $m$-chloroaniline) substantially reducing the electron density on the ring and leading to diminution in conjugation, lower chain length, and hence lower thermal stability.

\section{Morphological Studies}

Figure 4 presents SEM micrographs of copolymers OTMC11 and OTOC11. The SEM micrograph of PANI salt reveals sharp-edged granular particles with a lamellar structure on one side and a smooth pattern on the other. ${ }^{29}$ However, the copolymers had crystalline and amorphous morphologies. They also exhibited a pattern of sharp-edged particles like the PANI salt; the size of the particles decreased with increasing chloroaniline content in the copolymer, and concurrently increasing amorphous domains were also noticed. Copolymer OTMC11 was the most crystalline polymer. The $m$-toluidine/chloroaniline copolymers were generally more crystalline than the corresponding $o$-toluidine copolymers with chloroaniline. The increased crystallinity of the $m$-toluidine/ $m$-chloroaniline copolymers was also substantiated by their higher conductivity, which was an order of magnitude higher than that of homopolymer PMT.

Detailed studies on the crystallinity of various PANIs have been reported. ${ }^{30}$ The crystallinity of a conductive polymer is known to be affected by the method of synthesis, substitution, protonation, and so forth. ${ }^{31}$ The positions of the Bragg peaks and their $d$ values, obtained from the X-ray 


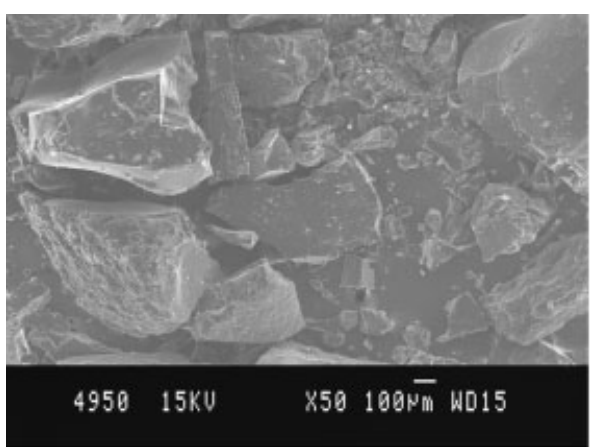

(a)

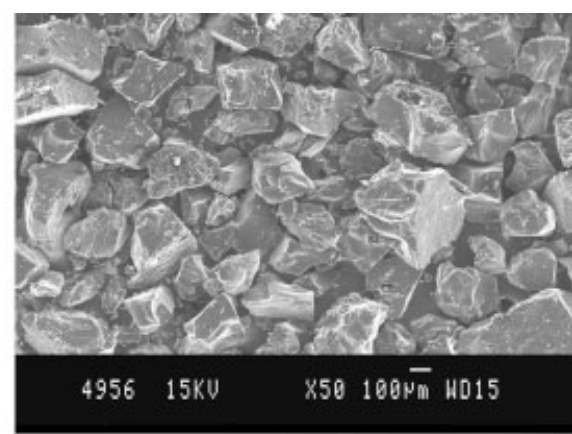

(b)

Figure 4. SEM images of (a) OTMC11 and (b) OTOC11.

powder diffractograms, are given in Table 3 . The copolymers exhibited their strongest peak at $2 \theta$ $=9-10^{\circ}$, their second strongest peak at $23-26^{\circ}$, a medium peak at $19-22^{\circ}$, and a weak peak at $14-16^{\circ}$. The number of diffraction peaks in the wide-angle X-ray diffractogram decreased with increasing chloroaniline content in the copolymer. These results indicate that the addition of the chloroaniline unit to PANI increased the intermolecular chain spacing and the amorphous nature. Copolymer OTMC11, showing the maximum number of sharp peaks in the XRD patterns, was the most crystalline copolymer, and this was consistent with the SEM studies. The X-ray powder diffraction patterns of the copolymers agreed with the results deduced from the SEM studies regarding the semicrystalline and amorphous nature of the copolymers.

\section{Solubility}

All $m$-toluidine $/ m$-chloroaniline copolymers, except for MTMC31, were completely soluble in polar solvents such as DMSO, dimethylformamide (DMF), and $N$-methylpyrrolidone (NMP) and par- tially soluble in less polar solvents such as chloroform. However, the copolymers of $o$-toluidine with higher $o$-chloroaniline contents, OTOC11 and OTOC12, were completely soluble. $m$-Chloroaniline copolymers had increased solubility in comparison with that of the $o$-chloroaniline ones. For example, copolymer OTMC11 was completely soluble in polar solvents, whereas OTOC11 was only partially soluble. Similarly, a copolymer of $m$-toluidine, MTOC11, was partially soluble, whereas MTMC11 was completely soluble in solvents such as DMSO, DMF, and NMP.

\section{CONCLUSIONS}

$o-/ m$-Chloroanilines were copolymerized with $o-/$ $m$-toluidines by chemical oxidative polymerization with ammonium persulfate as the oxidizing agent. These copolymers exhibited higher conductivities than the respective homopolymers, and this may be attributed to greater electron delocalization along the polymer chain due to the donoracceptor relations between the methyl group of toluidine and the chloro group of chloroaniline.

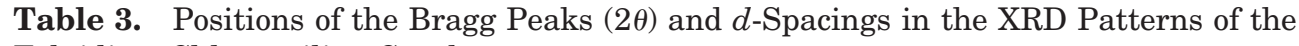
Toluidine-Chloroaniline Copolymers

\begin{tabular}{|c|c|c|c|c|c|c|c|}
\hline Sample & & & & $2 \theta[d(\AA)]$ & & & \\
\hline OTOC21 & $8.31(10.63)$ & $9.63(9.18)$ & $10.5(8.42)$ & $18.33(4.84)$ & $19.46(4.56)$ & $24.9(3.57)$ & \\
\hline OTOC11 & $8.9(9.93)$ & & & $18.89(4.69)$ & $21.27(4.17)$ & $24.22(3.67)$ & $27.6(3.23)$ \\
\hline OTMC11 & $8.13(10.86)$ & $11.57(7.64)$ & $12.95(6.83)$ & $19.65(4.51)$ & $22.96(3.87)$ & $24.03(3.70)$ & $26.16(3.40)$ \\
\hline MTMC21 & 7.38 (11.97) & $8.13(10.86)$ & $13.20(6.70)$ & $14.45(6.12)$ & $20.09(4.42)$ & $24.85(3.58)$ & $33.55(2.67)$ \\
\hline MTMC11 & $7.88(11.21)$ & $8.38(10.86)$ & $10.52(8.40)$ & $16.16(5.48)$ & & $23.81(3.73)$ & $33.35(2.68)$ \\
\hline MTOC11 & $9.13(9.68)$ & $10.76(8.22)$ & $12.70(6.96)$ & & & $24.47(3.63)$ & $37.03(2.43)$ \\
\hline
\end{tabular}


The conductivity of these copolymers, which were prepared with various comonomer feed compositions, varied over a wide range of $10^{-1}$ $10^{-4} \mathrm{~S} / \mathrm{cm}$. The copolymers in their salt form were completely soluble in polar solvents such as DMSO, DMF, and NMP.

\section{REFERENCES AND NOTES}

1. Diaz, F. R.; Sánchez, C. O.; Del Valle, M. A.; Torres, J. L.; Tagle, L. H. Synth Met 2001, 118, 25.

2. Ng, S.-C.; Xu, L. Adv Mater 1998, 10, 1525.

3. Misra, R. A.; Dubey, S.; Prasad, B. M.; Singh, D. Indian J Chem Sect A 1999, 38, 141.

4. Liao, Y.-H.; Angelopoulous, M.; Levon, K. J Polym Sci Part A: Polym Chem 1995, 33, 2725.

5. Prévost, V.; Petit, A.; Pla, F. Synth Met 1999, 104, 79.

6. Rivas, B. L.; Sánchez, C. O.; Bernede, J.-C.; Basaez, L. J Appl Polym Sci 2003, 90, 706.

7. Kang, D.-P.; Yun, M.-S. Synth Met E 1989, 29, 343.

8. Wang, S. L.; Wang, F. S.; Ge, X. H. Synth Met 1986, 16, 99.

9. Wei, Y.; Hariharan, R.; Patel, S. A. Macromolecules 1990, 23, 758.

10. Leclerc, M.; Guay, J.; Dao, L. H. Macromolecules 1989, 22, 649.

11. Salavagione, H. J.; Acevedo, D. F.; Miras, M. C.; Motheo, A. J.; Barbero, C. A. J Polym Sci Part A: Polym Chem 2004, 42, 5587.

12. Karyakin, A. A.; Maltsev, I. A.; Lukachova, L. V. J Electroanal Chem 1996, 402, 217.

13. Fan, J.; Wan, M.; Zhu, D. J Polym Sci Part A: Polym Chem 1998, 36, 3013.

14. Having, E. E.; ten Hoeve, W.; Wynberg, H. Synth Met 1993, 55, 299.
15. Kertesz, M.; Choi, C. H.; Sun, G. Polym Prep 1998, 39, 76 .

16. Demanze, F.; Yassar, A.; Garnier, F. Macromolecules 1996, 29, 4267.

17. Lu, H.-F.; Chan, H. S. O.; Ng, S.-C. Macromolecules 2003, 36, 1543.

18. Ruckenstein, E.; Sun, Y. Synth Met 1995, 74, 107.

19. Savitha, P.; Sathyanarayana, D. N. J Polym Sci Part A: Polym Chem 2004, 42, 4300.

20. Trivedi, D. C. In Handbook of Organic Conductive Molecules and Polymers; Nalwa, H. S., Ed.; Wiley: Chichester, England, 1993; Vol. 2, p 505.

21. Kim, Y. H.; Foster, C.; Chiang, J.; Heeger, A. J. Synth Met 1989, 29, 285.

22. Roe, M. G.; Ginder, J. M.; Wigen, P. E.; Epstein, A. J.; Angelopoulous, M.; Macdiarmid, A. G. Phys Rev Lett 1988, 60, 2789.

23. Gruger, A.; Novak, A.; Regis, A.; Colomban, P. J Mater Sci 1994, 328, 153.

24. Tzou, K.; Gregory, R. V. Synth Met 1993, 53, 365.

25. Ping, Z. J Chem Soc Faraday Trans 1996, 92, 3063.

26. Silverstein, R. M.; Webster, F. X. Spectrometric Identification of Organic Compounds, 6th ed.; Wiley: New York, 1998.

27. Savitha, P.; Sathyanarayana, D. N. Polym Int 2004, 53, 106.

28. Anand, J.; Palaniappan, S.; Sathyanarayana, D. N. In Handbook of Organic Conductive Molecules and Polymer; Nalwa, H. S., Ed.; Wiley: Chichester, England, 1993; Vol. 2, p 573.

29. Rao, P. S.; Sathyanarayana, D. N.; Palaniappan, S. Macromolecules 2002, 35, 4988.

30. Laridhani, M.; Pouget, J. P.; Scherr, E. M.; Mac Diarmid, A. G.; Jozefowicz, A. E.; Epstein, A. J. Macromolecules 1992, 25, 4106.

31. Pouget, J. P.; Jozefowicz, M. E.; Epstein, A. J.; Tang, X.; Mac Diarmid, A. G. Macromolecules 1991, 24, 779. 